\title{
Review on Internet Addiction in Adolescent: Biomolecular, Hatha Yoga Intervention, COVID-19 Pandemic and Immune Systems
}

\author{
I Putu Belly Sutrisna', \\ Cokorda Bagus Jaya \\ Lesmana ${ }^{2}$, \\ I Made Jawi ${ }^{3}$, \\ I Wayan Suka Yasa ${ }^{4}$, \\ I Gusti Bagus Wirawan ${ }^{4}$
}

'Department of Psychiatry, Wangaya General Hospital, Bali, Indonesia

${ }^{2}$ Department of Psychiatry, Faculty of Medicine, Udayana University, Bali, Indonesia

${ }^{3}$ Department of Pharmacology, Faculty of Medicine, Udayana University, Bali, Indonesia

${ }^{4}$ Department of Hindu Philosophy, Indonesian Hindu University, Bali, Indonesia

\begin{abstract}
Internet addiction in adolescent during pandemic COVID-19 will be predicted to be increased more than ever. There is need prevention or intervention program to solve this problem. Hatha yoga is considered to be impact mind-body stress relieving exercise found that improve the internet addiction sign and symptoms, but lack of evidence reviews about neurobiology model that effect Hatha yoga and internet addiction. This present review summarizes about Hatha yoga epigenetic mechanism effects could improve in internet addiction. PubMed, ResearchGate and Google scholar have been searched with the following key terms "Internet Addiction and Yoga", "Yoga and Mental Health", "Addiction", "Yoga", "Addiction and COVID-19", "exercises and mental health", "exercise and brain neuroplasticity" from December 2019 up to December 2020. Study in the internet addiction found that significant the expression level of the mRNA of D5 dopamine receptor was significant downregulated in peripheral blood lymphocytes (PBLs). The literature summarized in this review have shown that Hatha yoga effect through epigenetic process could improve the gene expression mRNA of D5 dopamine receptor in PBLs in the brain in order to improve internet addiction symptoms and sign and active transcription factor Nrf2 to increase the immune system.
\end{abstract}

Keywords: internet addiction disorder, behavior, addictive, RNA, messenger

\section{Introduction}

Internet become a part of human daily life in last twenty years. The internet has much positive impact for many people but there is also have negative impact such as internet addiction. The firsttime case of internet addiction was found at 1996 and until today there is need to improve how to prevent and interfere the internet addiction. In Diagnostic and Statistical of Mental Disorders, 5th edition (DSM-5), the internet addiction was included in section III that need more future research. ${ }^{1}$

In January 2020, WHO announced the outbreak of the COVID-19 as a health emergency of international concern declared as pandemic. ${ }^{2}$ The first step to preventing the spread of infectious diseases is quarantine and the effect of these condition found about two-thirds of the person reported using internet more during this pandemic. ${ }^{3}$ They used the internet to reduce stress and anxiety or to alleviate their mood.

Internet addiction pose risks to youth's mental health and produce negative consequences in everyday life like social withdrawal, loss of control internet use, feeling anger, symptom of distress and family conflict. Internet addiction become serious problem all over the world and need emergency for prevention and intervention strategy effectively. ${ }^{4}$ Intervention target for internet addiction base on biomolecular pathogenesis model. There is no evidence base treatment for internet addiction and still need further research. ${ }^{5}$

In pandemic COVID-19 era, WHO has established general health guidelines for staying at home period include physical activity and mental health. ${ }^{6}$ Yoga is one of physical activity that can do during pandemic COVID-19. Study pretest and posttest design, used Kimberly Young Internet Addiction Test, yoga was found significantly reduced a sign of internet addiction. ${ }^{7}$ In this article will be explain about how Hatha yoga as one as psychical activity can reduce internet addiction according by explanatory in biomolecular process model in order to find the alternative strategy for prevention and intervention internet addiction.
Dr. dr. Cokorda Bagus Jaya Lesmana, SpKJ.

Department of Psychiatry, Faculty of Medicine, Udayana University

Jl. Kesehatan I, Denpasar 80II4

Bali, Indonesia

Email: yaya@cokorda.com 


\section{Internet addiction: History and Now}

Internet addiction started launch from 1998, where that were more than 600 cases people whose uncontrol of internet usage and had relationship problem, academic problem, financial problem and loss of occupations. After this event, there were started a lot of research to think about internet addiction.

All the people generation in the world had accept that the internet has changed their world in ways both tiny and immense, especially children whose growing up today can't even imagine a world without the internet. Scientist began working to find a definition for internet addiction using measurable criteria. The forms of both substance and behavioral addiction such internet addiction looked have many similarities and identifying internet or technology addicts required the following traits: compulsive behavior, overuse of the technology, problem caused by overuse including interpersonal and health issues, time management challenges due to overuses of technology, tolerance requiring increased use and withdrawal when not using the technology.

\section{Internet addiction and adolescent}

More than $80 \%$ adolescent population can access to the internet in Asia, Europe and America and 45-55\% have been found in South America. ${ }^{1}$ Adolescent in 12-18 years old prefer want to be free from their parents and want to start with their peer group. They created own media social account and didn't want to their parents' control. There is an important need a good communication skill around children and parents to create digital diet and digital nutrition in order to get positive internet impact.

The expression of distress during adolescent tend to occur psychosomatic symptoms, psychomotor instability, boredom, fatigue, inclination to act and this trend towards action in overuse of internet especially when self-regulatory strategies are lacking and caregivers do not provide. Several studies have shown that internet addiction made sleep disturbance, overweight or obesity, frustration, depression, anxiety, attention-deficit/hyperactivity (ADHD), smoking problem, isolation, tendencies to suicidal behaviors, etc. ${ }^{9}$

\section{Internet addiction instrument}

Internet Addiction Test from Dr. Kimberly Young is the most used instrument in the western part of the world.9 This instrument already validating test in any language like French, German, Norwegian, Iranian, Chinese, Italian, and Indonesian. This instrument especially created to explore maladaptive behavior effect from over use of the internet which utilizes dependency criteria to formulate the diagnosis compose od 20 items on a 5-point Likert scale. The other one is the Chen Internet Addiction Scale, specifically designed to screen internet addiction in adolescent, which composed of 26 items on 4 points Likert scale. ${ }^{1}$

\section{Neurotransmitter Dopamine and \\ Reward System in internet addiction}

Neurotransmitters are the communicators the or messengers in the brain, via transmit information from neuron to neuron. It tells the brain to perform certain task. Additive behavior manipulates the brain neurotransmitters, become disruption in the brain. Dopamine is one of the neurotransmitters in the brain that the most important neurotransmitter at play in internet addiction. Dopamine is a natural drug in the brain when we experience something that feels good. That dopamine goes directly to nucleus accumbent, a cluster of nerve cells often called the brain pleasure center. After the dopamine neurotransmitter achieved the nucleus accumbent and we feel good, then the hippocampus records that feel-good experience as memory. The amygdala creates a condition response that makes we want to do that again. ${ }^{8}$

The internet can stimulate the pleasure center as sexual activity is and potentially more dangerous because the instant gratification rewards all day long compare with sex or food. We seek more and more dopamine to keep feeling good through technology. There are three dopamine pathways in the brain that might affected by changes in dopamine levels relate of reward. The first is the mesocortical pathway, linked with cognitive and emotional abilities as well as memory, attention, and how to learn. The second is nigrostriatal pathway, about movement and sensory stimulation, the third is mesolimbic pathway, linked the pleasure-seeking and reward desiring. ${ }^{8}$

\section{Molecular Biology of Internet Addiction}

Neuroscience found that thin anatomy structure and function abnormality pre frontal cortex (PFC) region in functional MRI in internet addiction patient. This brain area function for making decisions and impulse control, so in a internet addiction, this task will be impaired. Adolescent with Internet Gaming Disorder (IGD) have a decrease thickness than control in the orbitofrontal cortex (OFC), the insula, and the entorhinal cortex. This structural finding with an impaired cognitive control, decrease ability to voluntary drive their own internet activities. In other hand, there is increased cortical thickness observed in the inferior or middle temporal cortices, with associated with visual imagery and memory, and have been related to craving as induce in addicts by drug-related cues. In other studies, Internet addiction had less grey matter in anterior cingulate cortex (ACC), posterior cingulate cortex (PCC), dorsolateral $\mathrm{PFC}, \mathrm{OFC}$, and insula that involved cognitive control drive and impulse. The PCC on the other hand is implicate in attention and focusing of executive process. So, this condition make in internet addiction which exhibit a diminished efficiency of inhibition process and associated with impulsivity. ${ }^{1}$

Dopamine hyperstimulation in internet addiction make the brain function unstable when try to making decision and become impulsive. Adolescent fee 1 euphoria when using the internet 
because of a lot of dopamine release in the brain. This event will make the brain insensitive with dopamine receptor (tolerance), and making the dose of internet become more and more to make feel euphoria than before. ${ }^{10}$

\section{Internet Addiction Preventing and Treatment During COVID-I 9}

The changes of lifestyle in pandemic COVID-19 become increasing the risk of internet addiction. Applying education or virtual counseling programs in order to reduce psychological problem of internet addiction and provide solutions can be helpful. ${ }^{11}$ Engaging in physical activity regularly is need to keep a healthy body and boosting mood by reducing levels of stress hormones, stimulating the production of endorphins and having beneficial effect on immune function.

Intervention of internet addiction can be focus on parent and individual side. From individual side, a common treatment is cognitive behavior therapy. ${ }^{10}$ Beside cognitive behavior therapy, pharmacology treatments also can be addressed. ${ }^{12}$ Multi modal counselling and motivation interviewing have been prove to be effective but there is no long-term longitudinal studies have been yet performed nor large epidemiological data are available.

\section{Internet Addiction in Adolescent and}

\section{Hatha Yoga}

Yoga is one as integrative therapy approach to treat addiction. The therapeutic effects of yoga are widely studied and found it could decrease the symptoms of internet addiction. In the western world, the most common aspect of yoga are the physical postures and breathing exercise of Hatha Yoga and meditation. The basic concept of yoga reveal that the human body is integrative system of various interrelated and inseparable dimension. Yoga practice enhance body flexibility and muscular strength, improve respiratory and cardiovascular function and overall well-being. ${ }^{13}$ There was one studied found that yoga had significantly reduced from $90 \%$ to $99 \%$ recover internet addiction symptoms in moderate and mild case. The implication of yoga will have immense physical, emotional and spiritual well-being and maintenance physical and mental health.

Yoga is an integrated mind-body practice, originating about 5000 years BC in ancient India. ${ }^{14}$ The most common form of yoga practice in the west is Hatha yoga, which focus on breathing technique, physical posture (asanas) and meditation. The Hatha yoga is more physical posture in nature compare with the other yoga. ${ }^{15}$ Yoga is one of the aerobic exercises that asanas posture might increase oxygen blood flow to the brain to repair hyperdopaminergic and neuroplasticity via brain-derived neurotropic factor (BDNF).

\section{Hatha Yoga Exercise and Immune System}

The immune system is a critical homeostatic system that destroys foreign agent in human's body. Hatha yoga practice did not primarily evolve as a system of physical exercise, but also as a healing system which is involve immunology system. Yoga modulates the central stress response and to regulate autonomic balance that may impact immune functions. General pattern emerged that yoga can down-regulate pro-inflammatory markers. The study revealed decrease in IL-1 $\beta$, as well as indication for reduction in IL-6 and TNF-alpha, that might beneficial effects by enhancing cell-mediated and mucosal immunity. ${ }^{14}$ Yoga exercise improve the human immune by an increase blood levels of natural killer cells, neutrophils and antibodies. ${ }^{17}$

\section{Hatha Yoga Exercise for Internet Addiction}

Sport psychology has suggested that the success or failure is usually characterized by the FITT principle, which contain Frequency (F)-how often an activity is done, Intensity (I)-how hard the activity is, Time (T)-the duration of activity, Type (T)the mode or kind of activity done. The FITT principle allows all of an exercise including Hatha yoga which is directly related to health benefit.

We suggest that Hatha yoga recommendation in adolescent doing three times frequency per week, with moderate intensity between 30 and $70 \%$ Maximum heart rate, and duration time in minimum 30 minutes. Hatha yoga is type of combine aerobic and anaerobic exercise, and doing regularly in 8 weeks to get benefit effect in neuroplasticity from the brain and could change mood in internet addiction. ${ }^{18}$

\section{Conclusion}

The pandemic COVID-19 era could increase the evidence of internet addiction in adolescent. This review summarizes that Hatha yoga benefit for internet addiction and immune systems, but lack of scientific research explores about neurobiological process about this mechanism.

\section{References}

1. Cerniglia L, Zoratto F, Cimino S, Laviola G, Ammaniti M, Adriani W. Internet Addiction in adolescence: Neurobiological, psychosocial and clinical issues. Neuroscience and Biobehavioral Reviews. 2017;76(A):174-184. 
2. Ryalino C. Covid-19: What we know so far. Bali J Anaesthesiol 2020;4(1):1-2. doi 10.4103/BJOA.BJOA_17_20

3. Jovic J, Pantovic-Stefanovic M, Mitkovic-Voncina M, Dunjic-Kostic B, Mihajlovic G, Milanovic S, et al. Internet use during coronavirus disease of 2019 pandemic: Psychiatric history and sociodemographics as predictors. Indian J Psychiatry. 2020;62(Suppl 3):S383-S390.

4. Zhu Y, Zhang H, Tian M. Molecular and functional imaging of internet addiction. Biomed Res Int. 2015;2015:378675. doi: $10.1155 / 2015 / 378675$

5. Greenfield DN. Treatment Considerations in Internet and Video Game Addiction: A Qualitative Discussion. Child Adolesc Psychiatr Clin N Am. 2018;27(2):327-344. doi: 10.1016/j.chc.2017.11.007.

6. Ryalino C. How indonesia copes with coronavirus disease 2019 so far (part two): Is the country ready for the new norm?. Bali J Anaesthesiol 2020;4(3):89. doi: 10.4103/BJOA.BJOA_108_20

7. Ghassemzadeh L, Shahraray M, Moradi A. Prevalence of internet addiction and comparison of internet addicts and non-addicts in Iranian high schools. Cyberpsychol Behav. 2008;11(6):731-3. doi: 10.1089/cpb.2007.0243.

8. Kristiana S, Enjeline H, Thung SL, Hans C, Adrian A, Putri SL, et al. The Impact of Physical Distancing and Associated Factors Towards Internet Addiction Among Adults in Indonesia During COVID-19 Pandemic: A Nationwide Web-Based Study. Frontiers in Psychiatry. 2020;11:924 doi: 10.3389/fpsyt.2020.580977

9. Marin MG, Nuñez X, de Almeida RMM. Internet Addiction and Attention in Adolescents: A Systematic Review. Cyberpsychol Behav Soc Netw. 2021;24(4):237-249. doi: 10.1089/cyber.2019.0698

10. Liu M, Luo J. Relationship between peripheral blood dopamine level and internet addiction disorder in adolescents: a pilot study. Int J Clin Exp Med. 2015;8(6):9943-9948.
11. Eidi A, Delam H. Internet addiction is likely to increase in home quarantine caused by coronavirus disease 2019 (COVID 19). Journal of Health Sciences \& Surveillance System. 2020;8(3):136-137. doi: 10.30476/jhsss.2020.87015.1104

12. Bisen SS, Deshpande YM. Understanding internet addiction: a comprehensive review. Mental Health Review Journal. 2018;23(3):165-184. doi: 10.1108/MHRJ-072017-0023

13. Tripathi A. Impact of Internet Addiction on Mental Health: An Integrative Therapy Is Needed. Integrative Medicine International. 2018;4(3):215-222. doi: 10.1159/000491997

14. Falkenberg RI, Eising C, Peters ML. Yoga and immune system functioning: a systematic review of randomized controlled trials. Journal of Behavioral Medicine. 2018;41(4):467-482. doi: 10.1007/s10865-018-9914-y

15. Khanna S, Greeson JM. Therapies for Addiction. A Narrative Review of Yoga and Mindfulness as Complementary Therapies for Addiction. 2014;21(3):244252. doi: 10.1016/j.ctim.2013.01.008.A

16. Petzinger GM, Holschneider DP, Fisher BE, McEwen S, Kintz N, Halliday M, et al. The Effects of Exercise on Dopamine Neurotransmission in Parkinson's Disease: Targeting Neuroplasticity to Modulate Basal Ganglia Circuitry. Brain Plasticity. 2016;1(1):29-39. doi: 10.3233/bpl-150021

17. da Silveira MP, da Silva Fagundes KK, Bizuti MR, Starck É, Rossi RC, de Resende E Silva DT. Physical exercise as a tool to help the immune system against COVID-19: an integrative review of the current literature. Clin Exp Med. 2021;21(1):15-28. doi:10.1007/s10238-020-00650-3

18. Mandolesi L, Polverino A, Montuori S, Foti F. Effects of Physical Exercise on Cognitive Functioning and Wellbeing : Biological and Psychological Benefits. 2018;9(4):1-11. doi: 10.3389/fpsyg.2018.00509 